\title{
Investigation of the Carbon Nanotubes Functionalization Effect on the Composite Material Conductive Properties
}

\author{
R.R. Garipov ${ }^{1}$, S.M. Khantimerov ${ }^{1}$, N.M. Suleimanov ${ }^{1,2}$ \\ ${ }^{1}$ Zavoisky Physical-Technical Institute, FRC Kazan Scientific Center of RAS, 10/7, Sibirsky tract, Kazan, 420029, Russia; \\ ${ }^{2}$ Kazan State Power Engineering University, 51, Krasnoselskaya ul., Kazan, 420066, Russia \\ * Corresponding author. Tel.: + 790467315 45.E-mail: ranis@kfti.knc.ru
}

\begin{abstract}
The changes in the morphology of conical carbon nanotubes and composition of functional groups on their surface during liquid-phase functionalization in various solutions were investigated. The dependence of the type of functional groups on the composition of oxidizing agents was studied. The influence of the type of functional groups on the carbon nanotubes surface on the conductive properties of the polymer composite material based on them was established.
\end{abstract}

\section{Keywords}

Carbon nanotubes; functionalization; composite material; electrical conductivity.

(C) R.R. Garipov, S.M. Khantimerov, N.M. Suleimanov, 2020

\section{Introduction}

Due to their unique physicochemical properties and high aspect (length to diameter) ratio, carbon nanotubes (CNTs) are considered as promising fillers for various materials for the directed change in their mechanical and electrophysical properties [1]. It is known [2] that carbon nanotubes in its original form have a large surface energy and therefore form agglomerates. Agglomeration of CNTs leads to their irregular distribution in the material being modified. In addition, the graphene surface of the nanotube forms only weak van der Waals bonds with the material [3]. Thus, the interaction between the nanotube and the material is weak. The most effective way to solve this problem is the functionalization of CNTs, which involves the attachment of various active functional groups to the surface of nanotubes. On the one hand, this allows solving the problem of agglomeration of carbon nanotubes, and, on the other hand, to ensure the creation of covalent bonds between the nanotube and the material.

There are a large number of works devoted to the functionalization of CNTs [4-7]. However, in most papers, the properties of carbon nanotubes functionalized only under certain conditions using a certain type of oxidizing medium are considered. In addition, in the majority of works related to the study of composite materials based on functionalized carbon nanotubes and polymers, the effect of the functionalization of CNTs on the properties of such a material is not studied [8-11].

This work is devoted to investigation of carbon nanotubes functionalized by liquid-phase oxidation using various compositions of oxidizing agents. It is aimed to study the effect of functionalization on the electrical conductivity of a composite material based on them.

\section{Materials and Methods}

\section{Synthesis of multi-walled carbon nanotubes}

The synthesis of conical carbon nanotubes was carried out by chemical vapor deposition at the experimental facility created in our Institute. Granular polyethylene was used as a carbon source. The synthesis was carried out at $800^{\circ} \mathrm{C}$ in a helium atmosphere. In the process of synthesis CNTs of conical type were formed. Carbon nanotubes had a length of several microns, an outer diameter of 40-50 nanometers and the size of internal channels 
were from 9 to 20 nanometers. The interplane distance in such conical carbon nanotubes (cCNTs) was $0.34 \mathrm{~nm}$. The largest size of the inner channel correlatedto the size of nickel nanoparticles observed at the ends of some nanotubes. This type of nanotube consisted of joined conical segments with open ends, as described elsewhere [12]. In contrast to the more traditional, cylindrical, cCNT shave a greater number of broken chemical bonds, therefore, they can be more easily functionalized.

\section{Functionalization of carbon nanotubes}

Functionalization of conical carbon nanotubes was carried out by liquid-phase oxidation in various oxidizers in the ratio of $0.1 \mathrm{~g}$ of carbon nanotubes per $100 \mathrm{ml}$ of oxidizer. The treatment was carried out at a temperature of $70{ }^{\circ} \mathrm{C}$ for 1.5 hours under ultrasonic influence. Functionalized carbon nanotubes were rinsed with distilled water to neutral $\mathrm{pH}$ and dried under vacuum at $90{ }^{\circ} \mathrm{C}$ for 8 hours. Table 1 shows the compositions of oxidizing media.

\section{The study of functionalized carbon nanotubes}

The study of the structure of the initial and functionalized carbon nanotubes was carried out using Auger-electron spectrometer JAMP 9510F ("JEOL", Japan) at ultrahigh vacuum $\left(<10^{-9} \mathrm{~mm} \mathrm{Hg}\right)$.

The study of the surface state of functionalized carbon nanotubes was carried out by IR spectroscopy on the infrared (IR) Fourier spectrometer Vector-27 (Bruker), with an optical resolution of $4 \mathrm{~cm}^{-1}$ and the accumulation of 32 scans in the range $4000-400 \mathrm{~cm}^{-1}$.

Samples for the study were prepared in the form of tablets with KBr: ground in a mortar, and then pressed in a mold under a pressure of 10 atmospheres (the procedure with one tablet was repeated up to 10 times to achieve uniformity).

\section{Preparation of composite material samples and investigation of electrical properties}

To prepare samples of the composite material, functionalized cCNTs were dispersed in isopropyl alcohol in a ratio of $1 \mathrm{mg}$ of nanotubes per $10 \mathrm{ml}$ of isopropyl alcohol for 1 hour. The resulting dispersion was filtered to a paste state and injected into the epoxy resin. The mixing of carbon nanotubes with epoxy resin was carried out by a magnetic stirrer. Removal of isopropyl alcohol residues was carried out in a vacuum chamber. After removal of isopropyl alcohol, the resulting mixture was mixed with a theta hardener, degassed and poured into a $10 \times 10 \times 1 \mathrm{~mm}$ cell.

Table 1

\section{Names of samples, with a description of the composition of oxidants}

\begin{tabular}{cl}
\hline $\begin{array}{c}\text { No. } \\
\text { of samples }\end{array}$ & \multicolumn{3}{c}{ Method of treatment } \\
\hline 1 & $\begin{array}{l}\text { Initial cCNTs } \\
\text { Treatment in concentrated } \mathrm{H}_{2} \mathrm{O}: \mathrm{HNO}_{3} \\
\text { in } 1: 1 \text { ratio } \\
\text { Treatment in a solution of } \mathrm{H}_{2} \mathrm{O}: \mathrm{HCl} \text { in the ratio } \\
1: 1 \\
3\end{array}$ \\
& $\begin{array}{l}\text { Treatment in a solution of } \mathrm{H}_{2} \mathrm{O}: \mathrm{HNO}_{3}: \mathrm{HCl} \\
\text { in the ratio } 2: 1: 1 \\
\text { Treatment in a solution of } \mathrm{HNO}_{3}: \mathrm{H}_{2} \mathrm{O}_{2}\end{array}$ \\
& $\begin{array}{l}\text { in the ratio } 1: 1 \\
\text { Treatment in a solution of } \mathrm{HCl}_{2}: \mathrm{H}_{2} \mathrm{O}_{2} \\
\text { in the ratio } 1: 1\end{array}$ \\
&
\end{tabular}

The study of the conductive properties of the composite material was carried out by four probe method. To do this, electrodes were attached to the surface of the samples using silver paste.

\section{Results and Discussion}

Auger microscopy was used to establish morphological changes and fragmentation of carbon nanotubes after functionalization (Fig. 1).

Micrographs show that agglomerates break down to individual nanotubes during the treatment process of CNTs.

The original and functionalized samples of cCNTs were investigated by IR spectroscopy. Absorption bands due to the presence of $\mathrm{C}-\mathrm{H}$ bonds $(2933,2854$, $\left.800 \mathrm{~cm}^{-1}\right)$, hydroxyl $\left(3450 \mathrm{~cm}^{-1}\right)$, ketone $\left(1650 \mathrm{~cm}^{-1}\right)$ and ether $\left(1085 \mathrm{~cm}^{-1}\right)$ groups were detected on the IR spectra of the initial and functionalized samples (Fig. 2).

Absorption bands due to the presence of hydroxyl $(\mathrm{C}-\mathrm{O}-\mathrm{H})$, ketone $(\mathrm{C}=\mathrm{O})$ and ether $(\mathrm{C}-\mathrm{O}-\mathrm{C})$ groups are observed on the IR spectra of samples No. 2, No. 3 and No. 4. For samples No. 5 and No. 6, the same absorption bands are observed on the IR spectra, except for the absorption band caused by ether groups. The content of ether functional groups in these samples decreases, however, there is an increase in the content of hydroxyl and ketone functional groups $[13,14]$.

To establish the influence of the type of functional groups on the surface of the cCNTs on the conductive properties of the composite material, samples No. 4 and No. 5 were introduced into the polymer matrix. The choice of these samples is due to the fact that sample No. 4 contains more ester functional groups compared to other samples, and sample No. 5 is characterized by a low content of ester groups and a high content of hydroxyl and ketone groups. 


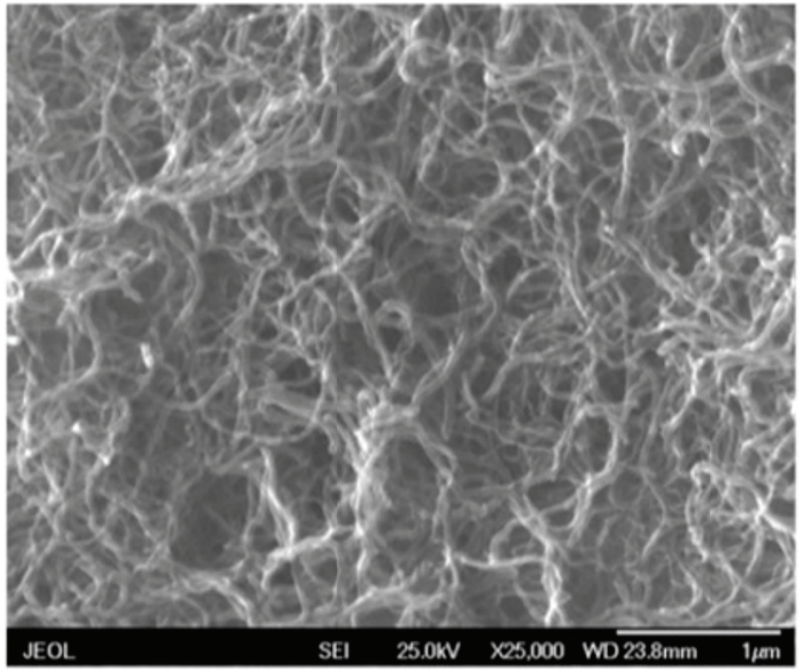

a)

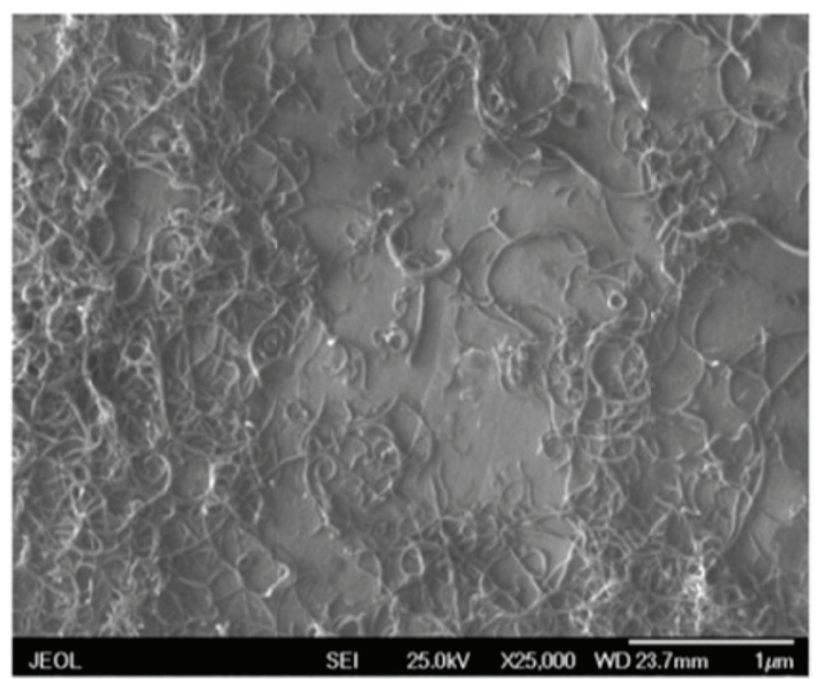

b)

Fig. 1. Electronic micrographs of the initial (a) and functionalized (b) samples of cCNTs

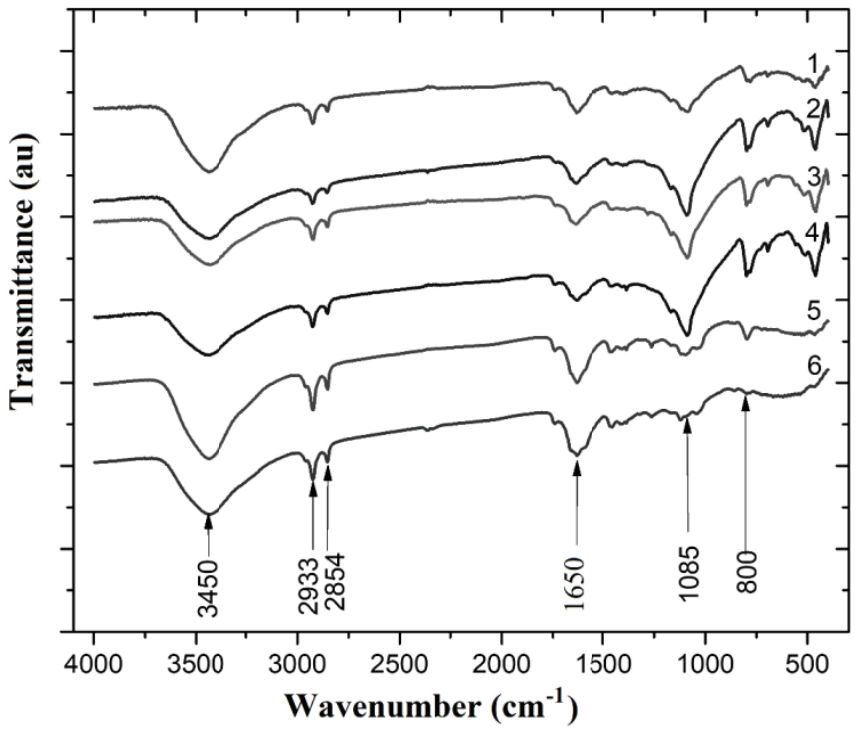

Fig. 2. IR spectra of initial and functionalized samples of cCNTs

The study of the conductive properties of the composite material was carried out by a four-probe method. Fig. 3 shows the concentration dependence of the conductivity of the composite material with cCNTs samples No. 4 and No. 5.

Figure 3 shows that the concentration dependence of the conductivity of the composite material is nonlinear. It is also obvious that the electrical conductivity of the composite based on MWNTs treated in a mixture of $\mathrm{HNO}_{3}: \mathrm{H}_{2} \mathrm{O}_{2}$ is $1.5-4$ times (at different concentrations)higher than that in sample No. 4. It is assumed that cCNTs containing hydroxyl and ketone functional groups, compared to cCNTs containing ester groups, are more compatible with the

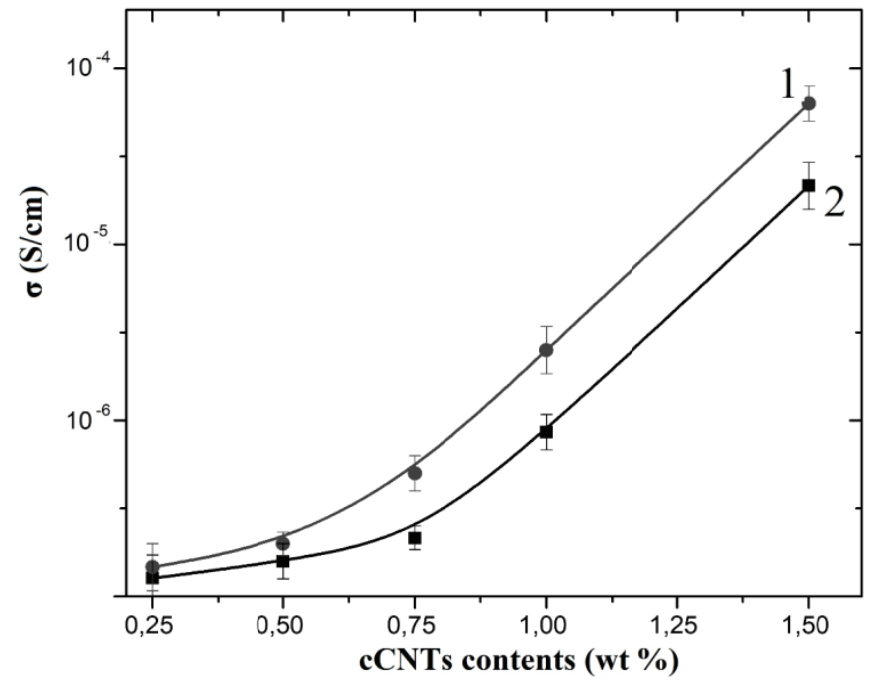

Fig. 3. Concentration dependence of electrical conductivity of composite material samples: 1 - sample No. $5 ; 2$ - sample No. 4

epoxy resin polymer matrix, resulting in more quantity of percolation channels.

\section{Conclusion(s)}

Changes in the morphology of conical carbon nanotubes and composition of functional groups on their surface during liquid-phase functionalization in various solutions were investigated. With Auger microscopy method it was shown that functionalization allows to break up the agglomerates to individual nanotubes. IR spectroscopy investigations showed that treatment even in aqueous acid solutions allows you to impart functional groups. The concentration 
dependence of the conductivity of a composite material based on epoxy resin and functionalized cCNTs with different types of functional groups was investigated by the four-probe method. It was shown that the concentration dependence of the electrical conductivity of the composite material has a nonlinear character, and the relationship between the functionalization of cCNTs and the electrical conductivity of the composite material on their basis was established.

\section{Acknowledgement(s)}

The study was conducted with the financial support of the RFBR and the Government of the Republic of Tatarstan in the framework of the scientific project No. 18-48-160021 $r$ a and the program of the Presidium of the RAS No. 8 .

\section{References}

1. Chen S., Corrias M., Wu G., Chen D., Jiang M. A new approach to the A new approach to the functionalization of single-walled carbon nanotubes with both alkyl and carboxyl groups. Chemical Physics Letters, 2005, 402, 312-317.

2. Buang N.A., Fadil F., Majid Z.A., Shahir S. Characteristic of Mild Acid Functionalizatization of multiwalled carbon nanotubes towards high dispersion with low structural defect. Digest Journal of Nanomaterials and Biostructures, 2012, 7(1), 33-39.

3. Shanmugharaj A.M., Bae J.H., Lee K.Y. Physical and chemical characteristics of multiwalled carbon nanotubes functionalized with aminosilane and its influence on the properties of natural rubber composites. Composites Science and Technology, 2007, 67, pp. 1813-1822.

4. Jeong T., Kim T.H., Kim W.-Y., Lee K.H., Hahn Y.-B. High yield purification of carbon nanotubes with $\mathrm{H}_{2} \mathrm{~S}-\mathrm{O}_{2}$ mixture. Korean Journal of Chemical Engineering, 2002, 19(3), 519-523.

5. Smith M.R., Hedges S.W., La Count R., Kern D., Shah N., Huffman G.P., Bockrath B. Selective oxidation of single-walled carbon nanotubes using carbon dioxide. Carbon, 2013, 41(6), 1221-1230.

6. Chaturvedi P., Verma A., Singh A., Chaudhary P.K., Harsh, Basu P.K. Carbon nanotube - purification and sorting protocols. Defence Science Journal, 2008, 58(5), 591-599.

7. Osorio A.G., Silveira I.C.L., Bueno V.L., Bergmann C.P. $\mathrm{H}_{2} \mathrm{SO}_{4} / \mathrm{HNO}_{3} / \mathrm{HCl}$-Functionalization and its effect on dispersion of carbon nanotubes in aqueous media. Applied Surface Science, 2008, 255, 2485-2489.

8. Kitano H., Tachimoto K., Anraku Y.J. Functionalization of single-walled carbon nanotube by the covalent modification with polymer chains. J. Colloid and Interface Science, 2007, 306, 28-33.

9. Song W., Zheng Z., Tang W., Wang X. A facile approach to covalently functionalized carbon nanotubes with biocompatible polymer. Polymer, 2007, 48, 36583663.

10. Kyotani T., Nakazaki S., Xu W., Tomita A. Chemical modification of the inner walls of carbon nanotubes by HNO oxidation. Carbon, 2001, 39, 771-785.

11. Xu T., Yang J., Liu J., Fu Q. Surface modification of multi-walled carbon nanotubes by $\mathrm{O}_{2}$ plasma. Applied Surface Science, 2007, 253, 8945-8951.

12. Khantimerov S.M., Togulev P.N., Kukovitsky E.F., Lyadov N.M., Suleimanov N.M. Effect of Electrochemical Treatment on Electrical Conductivity of Conical Carbon Nanotubes. Journal of Nanotechnology, 2016, 1-2, 1-5. doi: 10.1155/2016/8034985

13. Atieh M.A., Bakather O.Y., Al-Tawbini B., Bukhari A.A., Abuilaiwi F.A., Fettouhi M.B. Effect of carboxylic functional group functionalized on carbon nanotubes surface on the removal of lead from water. Bioinorganis Chemistry and Applications, 2010, 11,1-9.

14. Branca C., Frustery F., Magazu V., Mangione A. Characterization of Carbon Nanotubes by TEM and Infrared Spectroscopy. The Journal of Physical Chemistry B, 2004, 108(11), 3469-3473. 